\title{
NECESIDADES COMO CRITERIO FUNDAMENTADOR DE LOS DERECHOS HUMANOS*
}

\author{
NEEDS AS A FOUNDING CRITERION OF HUMAN RIGHTS \\ Yamid Enrique Cotrina Gulfo**
}

\begin{abstract}
RESUMEN: En el presente trabajo se propone una síntesis de las necesidades humanas condensadas en los pactos internacionales de derechos humanos (Derechos Civiles y Políticos y Derechos Económicos, Sociales y Culturales) al igual que en los mecanismos emergentes que reconocen los actuales retos en derechos humanos y condiciones de vida de las poblaciones como la Agenda 2030 - Objetivos de Desarrollo Sostenible. Relacionándola con la teoría de las necesidades expuestas por Maslow de aquellas compuestas por las de carácter radical de mantenimiento y de autorrealización. Se hace lo propio con los instrumentos internacionales de derechos humanos y una relación con lo propuesto en la doctrina jurídica.
\end{abstract}

ABSTRACT: This paper proposes a synthesis of the human needs condensed in the international covenants on human rights (Civil and Political Rights and Economic, Social and Cultural Rights) as well as in the emerging mechanisms that recognize the current challenges in human rights and living conditions of populations such as Agenda 2030 - Sustainable Development Goals. Relating it to the theory of the needs exposed by Maslow of those composed by those of radical character of maintenance and self-realization. The same is done with the international instruments of human rights and a relation with what is proposed in the juridical doctrine.

PALABRAS CLAVE: necesidades, derechos humanos, derechos civiles y políticos, derechos sociales.

KEYWORDS: needs, human rights, civil and political rights, social rights

Fecha de recepción: 06/04/2020

Fecha de aceptación: 06/04/2020

doi: https://doi.org/10.20318/universitas.2020.5520

\footnotetext{
* Trabajo presentado en el I Congreso de Alumnos y Alumnas del IDHBC.

** Estudiante de segundo curso del Máster en Estudios Avanzados en Derechos Humanos del IDHBC. E-mail: yaecog@hotmail.com
} 


\section{1.- NECESIDADES BÁSICAS O RADICALES ANALIZADAS DESDE LOS DERECHOS CIVILES Y POLÍTICOS}

Las necesidades de las personas son el faro hermenéutico seleccionado en esta investigación en aras de vincularlas a los derechos contemplados en el derecho internacional de los derechos humanos, lo que se propone a continuación es vincular tres niveles de necesidades según su nivel de prioridad a las tipologías de derechos contempladas según la práctica jurídica internacional. En primer orden se hace lo propio con los Derechos Civiles y Políticos y su relación con las necesidades básicas o radicales, desde una perspectiva finalista, esta clasificación de derechos tiene como objeto regular todas aquellas prestaciones jurídicas de las cuales el individuo es destinatario.

Siguiendo la línea teórica propuesta por Maslow, "las necesidades más básicas son defectivas, constituidas por un faltante y deben ser satisfechas para evitar una situación indeseable ${ }^{1 "}$. La cual, si es aplicable al ámbito de satisfacción relacionado con los derechos humanos, las necesidades más radicales son medibles con indicadores de cumplimiento que son absolutos, en términos de su cumplimiento y su incumplimiento. Son necesidades que son medibles según el resultado en términos de impacto de las políticas públicas de los Estados, puesto que solamente pueden ser satisfechas por las condiciones especificas de tales derechos.

Es por ello que exigen del criterio de idoneidad, propio de las normas jurídicas en términos de configuración normativa. Esto se explica en la especificidad que implica que algunas normas solo puedan ser reguladas una Ley e incluso, por un tipo de Ley específica, como es el principio de la reserva de Ley ${ }^{2}$, función reservada para ámbitos de carácter sensible como es la regulación de los derechos fundamentales o incluso, para garantizar su efectividad en el plano social.

Los indicadores para medir las necesidades insatisfechas derivadas de los derechos civiles y políticos pueden ser denominados como indicadores de cumplimiento o indicadores perfectos, toda vez que es posible un ejercicio reduccionista entre su cumplimiento e incumplimiento. La facilidad de medición en estos indicadores, obedece a que la mayoría de los preceptos contemplados en este Pacto Internacional, obedecen a prestaciones jurídicas de carácter fundamental, reconocidas así por la mayoría de constituciones, al menos en el mundo occidental.

\footnotetext{
1 GRASSETTI TONELLI, Eduardo. Satisfacción de necesidades y modelo social. PSOCIAL, 2018, vol. 4, no 1, p. 17.

2 BACIGALUPO, Mariano. La aplicación de la doctrina de los «Limites Inmanentes»a los derechos fundamentales sometidos a reserva de limitación legal (A propósito de la sentencia del Tribunal Administrativo Federal alemán de 18 de octubre de 1990). Revista española de derecho constitucional, 1993, no 38, p. 297-315.
} 
En el caso de alguna vulneración en el cumplimiento de estos derechos, no debe tomarse por falta de cobertura en alguna prestación. Sino por la aplicación indebida por causa de la omisión de un enfoque en cuando a las particularidades de la persona o grupo de personas afectados con la norma ejecutada. No obstante, este precepto no debe ser tomado por absoluto, en los criterios formales, en cuando a la inclusión en el ordenamiento jurídico interno de un país respecta. En aras de dar un abordaje completo al objeto de investigación, también deben ser tenidos en cuenta los factores materiales de los derechos.

Un claro ejemplo de lo anteriormente dicho se contempla en el derecho a la igualdad, si bien esta puede estar consagrada en las normas constitucionales de los ordenamientos jurídicos internos, puede verse sujeto el ejercicio de este derecho a diversos condicionamientos de carácter material. Esto puede verse representado en los siguientes aspectos: Igualdad ante la Ley y la prohibición de la discriminación directa, en cuanto a los criterios formales del derecho a la igualdad y la igualdad material en la igualdad de oportunidades, que puede verse aplicada en la discriminación indirecta y los ajustes razonables; las medidas de igualación positiva y las acciones positivas moderadas ${ }^{3}$. Las anteriores medidas en algunos casos pueden ser analizadas según los recursos de amparo impetrados ante las altas cortes de constitucionalidad de cada uno de los Estados o en los bloques regionales de derechos humanos en su jurisprudencia como es el caso de la Corte Interamericana de Derechos Humanos y el Tribunal Europeo de Derechos Humanos.

Los derechos contenidos en el Pacto Internacional de Derechos Civiles y Políticos, pueden ser clasificados según su impacto en individuales y colectivos.

\section{Tabla 1. Clasificación de Derechos Civiles y Políticos en su finalidad4}

\begin{tabular}{|l|l|}
\hline $\begin{array}{l}\text { Ámbito de } \\
\text { aplicación }\end{array}$ & Artículo del Pacto de Derechos Civiles y Políticos \\
\hline \multirow{4}{*}{$\begin{array}{l}\text { Derechos } \\
\text { individuales }\end{array}$} & $\begin{array}{l}\text { Art. 2. Derecho a contar con recursos legales ante vulneración de } \\
\text { derechos. }\end{array}$ \\
\cline { 2 - 2 } & Art. 3. Igualdad entre hombres y mujeres. \\
\cline { 2 - 2 } & Art. 6. Derecho a la vida y a la supervivencia. \\
\cline { 2 - 2 } & Art. 7. Prohibición de tratos inhumanos o degradantes. \\
\cline { 2 - 2 } & Art. 8. Prohibición de la esclavitud y servidumbre. \\
\cline { 2 - 2 } & Art. 9. Prohibición frente al arresto o detención arbitraria y \\
\hline
\end{tabular}

3 CAMPOY CERVERA, Ignacio. "Una revisión de la idea de dignidad humana de los valores de libertad, igualdad y solidaridad en relación con la fundamentación de lo derechos", Anuario de filosofía del derecho, No. 21, 2004, pp, 143-166.

4 Fuente: Con información de: Consejo de Europa, en línea: https://www.coe.int/es/web/compass/the-international-covenant-on-civil-andpolitical-rights 


\begin{tabular}{|c|c|}
\hline & Derecho a la seguridad personal. \\
\hline & Art. 10. Trato humano a las personas privadas de la libertad. \\
\hline & $\begin{array}{l}\text { Art. 11. Prohibición de condena por incumplimiento de } \\
\text { obligaciones. }\end{array}$ \\
\hline & Art. 12. Derecho a la libertad de movimiento. \\
\hline & $\begin{array}{l}\text { Art. 13. Prohibición de expulsión a extranjeros sin agotar el } \\
\text { debido proceso. }\end{array}$ \\
\hline & Art. 14. Igualdad ante la Ley y garantía de un debido proceso. \\
\hline & Art. 15. Irretroactividad de la Ley en materia penal. \\
\hline & Art. 16. Derecho a la personalidad jurídica \\
\hline & Art. 17. Libertad de pensamiento, conciencia y religión. \\
\hline & Art. 18. Libertad de opinión y expresión. \\
\hline & Art. 23. Derecho al matrimonio y a fundar una familia. \\
\hline & $\begin{array}{l}\text { Art. } 24 \text {. Derecho a la no discriminación, nacionalidad y } \\
\text { reconocimiento ante la Ley de los niños. }\end{array}$ \\
\hline & Art. 25. Derecho a la participación política. \\
\hline & Art. 26. Derecho a la igualdad y a la no discriminación. \\
\hline & Art. 1. Derecho a la autodeterminación de los Estados. \\
\hline Derechos & $\begin{array}{l}\text { Art. 20.Prohibición de la propaganda que promueva la guerra o el } \\
\text { odio nacional, racial o religioso. }\end{array}$ \\
\hline colectivos & Art. 21. Derecho de reunión. \\
\hline & Art. 22. Libertad de asociación \\
\hline & Art. 27. Derecho de las minorías religiosas, étnicas o lingüísticas. \\
\hline Atribuciones & Art. 4. Derecho de suspensión de los efectos del tratado. \\
\hline $\begin{array}{l}\text { comunidad } \\
\text { internacional }\end{array}$ & Art. 5. Criterios de interpretación del tratado. \\
\hline
\end{tabular}

Como puede apreciarse, una de las características esenciales de los Derechos Civiles y Políticos es la formación de límites del estado frente a los ciudadanos, asumidos por Dworkin como límites frente al poder político, la efectividad social de los derechos fundamentales ${ }^{5}$. La libertad negativa es entonces "la ausencia de obstáculos, barreras o restricciones, en la medida que se tiene la posibilidad de acción en sentido negativo"6. De igual manera "se predica del individuo en tanto toma decisiones que guían su vida o se identifica con ciertos estados y acciones suyas, (...) es una de las formas de concebir el autogobierno. Por su parte, la idea central detrás de la autonomía como no interferencia es la de un derecho del sujeto: un derecho a que otros se abstengan de determinadas acciones ${ }^{7 "}$. Es decir, en la medida que el Estado cede libertad a los ciudadanos, disminuye si campo de acción e intervención. Es por ello que los indicadores en este caso son de cumplimiento y absolutos por que se miden en el cumplimiento del principio de la no intervención del Estado en los ámbitos definidos en el Pacto Internacional.

\footnotetext{
5 DWORKIN, Ronald, "Los derechos en serio", Ariel Derecho, Madrid, 1989.

6 CARTER, Ian. "Libertad negativa y positiva. Astrolabio: revista internacional de filosofía", 2010, no 10, p. 15-35.

7 IOSA, Juan. "Libertad negativa, autonomía personal y constitución", Revista Chilena de Derecho, vol. 44 No 2, 2017, p, 497.
} 
La ratificación del pacto internacional mencionado implica compromisos en Derechos Humanos que adquieren los Estados y mediante los indicadores se contempla un instrumento de medición y evaluación en la pertinencia de las políticas públicas expedidas para garantizar el goce efectivo de derechos fundamentales. Para determinar la eficacia social del derecho además de tener en cuenta de la validez y legitimidad del derecho y los factores de eficacia jurídica de carácter instrumental como las agencias de información y control: tal es el caso de los datos obtenidos mediante informes, datos y estadísticas (PNUD: Programa de las Naciones Unidas para el Desarrollo; CEPAL: Comisión Económica para América Latina y el Caribe y ONGd: Organizaciones No Gubernamentales para el Desarrollo8.

Tales instrumentos resultan de carácter auxiliar para la formulación de políticas públicas pertinentes para la satisfacción de necesidades básicas vinculadas a los Derechos Fundamentales relacionados con la libertad y la dignidad de la persona, que actúan como fundamento o valor jurídico prevalente en la regulación de estos derechos que se consideran los más sensibles entre los fundamentales regulados en las cartas políticas de los países del hemisferio occidental.

\section{2.- NECESIDADES DE MANTENIMIENTO Y DERECHOS SOCIALES}

Contrastando la visión antropocéntrica individualista que predomina casi de manera absoluta en los derechos civiles y políticos y su relación con las necesidades radicales, se encuentra la interacción del individuo frente a sus congéneres en sociedad. Las necesidades sociales son aquellas que devienen de la interacción del individuo con sus pares en un nicho social ${ }^{9}$. Si se quiere asumir una visión económica de esta confluencia social, se puede centrar en como cada uno de los miembros de la sociedad aprovecha de manera común los recursos escasos que disponen ${ }^{10}$. Si por su parte, se asume desde una perspectiva axiológica, cuando existen puntos de encuentro que unen a todas estas personas en una sola identidad, esta población pasa a denominarse comunidad ${ }^{11}$.

El resultado de la interacción del individuo antes mencionada, como producto de la naturaleza social del ser humano, hace que

\footnotetext{
8 SORIANO, Ramón. "Sociología del Derecho", Ariel Derecho, Madrid: 1997.

9 PUIG LLOBET, Montserrat; SABATER MATEU, Pilar; ÁVILA RODRÍGUEZ, Nuria. Necesidades humanas: evolución del concepto según la perspectiva social. Aposta. Revista de Ciencias Sociales, 2012, no 54, p. 2.

10 MERCADOMALDONADO, Asael; GONZÁLEZ VELÁSQUEZ, Guillermo. La teoría del conflicto en la sociedad contemporánea. Espacios públicos, 2008, vol. 11, no 21, p, 200.

${ }^{11}$ GIMÉNEZ, Gilberto. Apuntes para una teoría de la identidad nacional. Sociológica México, 2015, no 21, p, 2.
} 
requiera ciertas habilidades, capacidades y destrezas para hacer frente al reto que representa su vida en sociedad. Cuando se sale de la esfera de lo eminentemente individual y se involucra al individuo en un colectivo de semejantes, surgen derechos que le sirven de prestaciones para poder hacer posible su vida en sociedad, entendida esta en el cumplimiento también de los deberes que tiene la persona para con su comunidad. Maslow definía estas necesidades también como aquellas de afiliación y afecto, en la que el individuo expresa su necesidad de relacionarse con los otros y ser parte de una comunidad, aplicadas estas al derecho las más destacables son las relacionadas con el derecho de asociación, participación y el de reunión ${ }^{12}$.

Lo anterior no solo se limita a la necesidad de interacción con los otros, también tiene que ver con derechos que solo pueden ser ejercidos mediante la intervención de otras personas, siendo la interdependencia una cualidad de las sociedades modernas. Cuando la persona necesita de sus semejantes para poder realizarse como individuo en su comunidad, se entiende el aspecto social de estos derechos que a su vez para su cumplimiento se requiere acatar deberes individuales para su realización.

Es por ello que "los derechos sociales son especialmente sensibles a las necesidades humanas, y como garantía de igualdad proporcionan razones para justificar una atención diferenciada en aquellos supuestos en que las desigualdades derivan de situaciones sobre las que las personas no tienen control. ${ }^{13 \prime}$ La igualdad es medible entonces con respecto a la relación que tiene con los otros, se es desigual en relación a sus semejantes, la igualdad es entonces el fundamento de los Derechos Económicos, Sociales y Culturales.

Estos indicadores son conocidos por ser imperfectos o de medición, puesto que su cumplimiento no depende de circunstancias absolutas. Los derechos sociales tienen la característica de que al propender por la igualdad en la sociedad pueden ser susceptibles de ser garantizados en mayor o menor medida entre unos y otros sectores. El acceso a oportunidades en condiciones de igualdad es lo que determina en fin último los indicadores de acceso a derechos sociales.

Frente a su realización presentan diversos desafíos, el principal de ellos es la libertad positiva que en si constituye garantizarlos, por necesitar una intervención del Estado. Siguiendo lo que expresó Isaías Berlin, mientras la libertad negativa es la "libertad de", la libertad positiva puede ser identificada como la "libertad para" enfocada hacia la autorrealización de la persona siguiendo los

12 MASLOW, A.H."A theory of human motivation". Psychological Review, 50, 1943.370-396.

13 AÑón, María José. Ciudadanía social: La lucha por los derechos sociales. Cuadernos electrónicos de filosofía del derecho, 2002, vol. 6, p. 1-15. 
preceptos contemplados en el libre desarrollo de su propia personalidad ${ }^{14}$.

Los derechos contenidos en el Pacto Internacional de Derechos Económicos, Sociales y Culturales, pueden ser clasificados según su impacto de la siguiente manera.

\section{Tabla 2. Clasificación de Derechos Económicos, Sociales y Culturales en su composición ${ }^{15}$}

\begin{tabular}{|c|c|}
\hline Composición & $\begin{array}{l}\text { Artículo del Pacto de Derechos Económicos, Sociales y } \\
\text { Culturales }\end{array}$ \\
\hline \multirow{14}{*}{$\begin{array}{l}\text { Parte } \\
\text { dogmática }\end{array}$} & Art. 1. Derecho a la libre autodeterminación de los pueblos. \\
\hline & Art. 3. Igualdad entre hombres y mujeres. \\
\hline & Art. 4. Principio de legalidad e interés general. \\
\hline & Art. 15. Derecho a participar en la vida cultural. \\
\hline & Art. 6. Derecho al trabajo. \\
\hline & Art. 7. Goce de condiciones equitativas en el trabajo. \\
\hline & Art. 8. Derecho de asociación sindical. \\
\hline & Art. 9. Derecho a la seguridad social. \\
\hline & Art. 10. Derecho a la protección de la familia. \\
\hline & Art. 11. Derecho a un nivel de vida adecuado. \\
\hline & Art. 12. Derecho a la salud física y mental. \\
\hline & Art. 13. Derecho a la educación. \\
\hline & Art. 14. Derecho a la enseñanza primaria gratuita y obligatoria. \\
\hline & Art. 15. Derecho a participar en la vida cultural. \\
\hline \multirow{3}{*}{$\begin{array}{l}\text { Garantías } \\
\text { para el goce } \\
\text { efectivo }\end{array}$} & Art. 2. Garantía de la efectividad de los DESC. \\
\hline & Art. 5,24 y 25 . Criterio de interpretación progresiva del pacto. \\
\hline & $\begin{array}{l}\text { Art. } 16,17,18,19,21,22 \text { y } 23 . \text { Deber de monitoreo y } \\
\text { evaluación por el ECOSOC. }\end{array}$ \\
\hline \multirow{5}{*}{$\begin{array}{l}\text { Parte } \\
\text { orgánica }\end{array}$} & $\begin{array}{l}\text { Art. } 26 \text { y } 30 \text {. Actores internacionales sujetos de firma y } \\
\text { ratificación. }\end{array}$ \\
\hline & Art. 27. Vigencia del tratado. \\
\hline & Art. 28. Jurisdicción del tratado. \\
\hline & Art. 29. Enmiendas al tratado \\
\hline & Art. 31. Idiomas oficiales del tratado. \\
\hline
\end{tabular}

Como puede apreciarse en el recuadro anterior, la inclusión recuadro de garantías adicionales a los criterios de interpretación del tratado, además del reconocimiento de derechos condicionados a la posibilidad institucional y presupuestaria de los Estados, hacen de estos derechos además de costosos, susceptibles de un cumplimiento según a su discrecionalidad fortaleciendo el margen de apreciación, no solo desde una perspectiva jurídica, sino también desde las realidades económicas en las que significa la asignación presupuestaria para la realización de estos derechos. El estado de bienestar, es entonces la realidad pretendida con esta declaración de

${ }^{14}$ FARRELL, Martín Diego. Libertad negativa y libertad positiva. Revista del Centro de Estudios Constitucionales, 1989, no 2, p. 9-20.

${ }^{15}$ Fuente: Con información del Consejo Económico y Social de las Naciones Unidas - ECOSOC: En línea:

https://www.ohchr.org/SP/ProfessionalInterest/Pages/CESCR.aspx 
derechos, pero los mecanismos por medio de los cuales se alcance no son de imperativo cumplimiento.

Finalmente, el goce efectivo de los derechos sociales está estrechamente vinculado al reto que representa en sí misma su justiciabilidad. La manera en como los Estados mediante sus instituciones hacen posible la realización de estas prestaciones morales juridificadas. Desde la teoría liberal clásica, se han desarrollado cinco argumentos ${ }^{16}$ contra esta pretensión de hacer realizables los derechos sociales:

1.- Imposibilidad del carácter subjetivo de los derechos sociales: Desde la definición más radical del positivismo, los derechos subjetivos no son más que facultades que tiene el individuo para exigirle al Estado el cumplimiento del deber jurídico contemplado ${ }^{17}$. De igual manera se afirma lo mismo desde su exigibilidad en los siguientes términos "los derechos sociales no pueden ser concebidos como derechos subjetivos, pues no configuran los elementos para merecer la forma de protección jurídicamente exigible que es propia de los derechos civiles y políticos ${ }^{18 "}$. Dada su naturaleza colectiva, los derechos sociales no pueden ser derechos subjetivos, toda vez que la naturaleza de estos, reside en la individualidad de la persona.

2.- La negativa a considerar a los derechos sociales como derechos positivos: El positivismo como paradigma jurídico ha sido el causante de la falta de protección de los derechos sociales y por lo tanto su efectividad sea puesta en entredicho. La resistencia que viene del positivismo se debe a que son normas jurídicas de carácter indeterminada, teniendo en cuenta la dimensión objetiva como un concepto iusfundamental al que muchos autores se refieren, pero pocos definen ${ }^{19}$. La aproximación jurídica más cercana, la hace Robert Alexy, cuando afirma que la faceta objetiva es el resultado de hacer una triple abstracción de la prerrogativa fundamental que elimine completamente su contenido jurídico-subjetivo. Es decir, se debe excluir, al titular del derecho, al sujeto obligado y la acción, situación o posición jurídica fundamental ${ }^{20}$. En este mismo sentido, Alexy ${ }^{21}$ y Böckenförde, justifican la existencia de las tres consecuencias de la calificación objetiva: El deber de protección, el efecto de irradiación o de expansión y la eficacia frente a terceros o la

16 ESPINO TAPIA, Diana Rocío. "Derechos sociales y justiciabilidad en la teoría constitucional de inicios del siglo XXI", Cuestiones Constitucionales: Revista Mexicana de Derecho Constitucional, Núm. 36. 2017, pp: 83-104.

17 CRUZ PARCERO, J., El lenguaje de los derechos. Ensayo para una teoría estructural de los derechos, Madrid, Trotta, 2007, pp. 71-76.

18 ATRIA, F., "¿Existen derechos sociales?", Discusiones, La Pampa, Universidad Nacional del Sur, año 4, núm.4, 2005, pp. 15-59.

19 TOLE MARTÍNEZ, Julián, La teoría de la doble dimensión de los derechos fundamentales en Colombia. El estado de cosas inconstitucionales, un ejemplo de su aplicación. Cuestiones Constitucionales, 2006, pp-253-316.

20 ALEXY, Robert, Teoría de los derechos fundamentales, trad. del alemán de E. Garzón Valdez, Madrid, Centro de Estudios Constitucionales, 1993.

${ }^{21}$ Ibid., pp, 138 y ss. 
eficacia jurídica objetiva de los derechos fundamentales ${ }^{22}$. La indeterminación de un sujeto pasivo de la obligación que supone el derecho consagrado en el derecho social hace que no sean más que aspiraciones con fines programáticos del Estado, por lo que su exigibilidad resulte imposible ${ }^{23}$.

3.- Los derechos sociales requieren de un fuerte presupuesto estatal para su ejecución: La libertad positiva requiere una acción por parte del Estado para garantizar las necesidades sociales, por lo que una inversión presupuestaria significativa desde las arcas públicas es imperiosa para lograr este cometido. Estos derechos tienen como finalidad el de promover la igualdad entre las personas, la igualdad como necesidad del individuo con clara implicación colectiva, hace que el hecho de expedir políticas públicas acordes a tal fin se vea amenazada por el factor de escasez de recursos que fue mencionado con anterioridad en la visión economicista de la relación del individuo y su grupo social, de ahí viene su protección debilitada. ${ }^{24}$

4.- El legislador goza de libre discrecionalidad en el desarrollo de los derechos sociales: Tal como se mencionó en la clasificación de los derechos consagrados en el Pacto de Derechos Económicos, Sociales y Culturales. En el contenido del tratado se hace mucho énfasis en la discrecionalidad que se le da a los Estados de hacer cumplir los términos establecidos en el contenido del tratado internacional, bien sea que por el hecho de que se traten de derechos costosos o también para hacerlo más atractivo para ser ratificado por la mayor parte de miembros de la comunidad internacional.

5.- La inexistencia de garantías jurisdiccionales para la efectividad de los derechos sociales: En el caso de los derechos más fundamentales contenidos en el pacto de Derechos Civiles y Políticos, se contiene la garantía de contar con recursos para el resarcimiento de derechos en el ordenamiento jurídico. Situación que en los Derechos Sociales no sucede, lo cual implica que la protección de estos derechos deba venir de la interpretación judicial de los órganos judiciales de cierre, en especial las cortes 0 tribunales constitucionales.

La interacción del individuo en sociedad hace que este tenga necesidades frente a los otros, que en el proceso de su inclusión social pueden ser consideradas necesidades sociales 0 de mantenimiento, que luego de haber alcanzado un mayor nivel en muchas de las necesidades contempladas en este ámbito de protección puede afirmarse que se encuentra en un estado de necesidades de mejora que implica la autorrealización del individuo.

\footnotetext{
22 BÖCKENFÖRDE, Ernst-Wolfgang, Escritos sobre derechos fundamentales, trad. de Juan Luis Requejo e Ignacio Villaverde, Baden-Baden, Nomos, 1993, p. 95.

${ }^{23}$ Ibid., $\mathrm{p}, 78$

24 PISARELLO, G., Los derechos sociales y sus garantías. Elementos para una reconstrucción, Madrid, Trotta, 2007, pp. 59.
} 
Lo cual, si se extrapola a nivel general, cuando los derechos sociales son alcanzados por una notable mayoría y su cobertura es de calidad, alcanzando las personas un nivel de vida adecuado en termino de mínimos aceptables, se analizaría el siguiente estadio en términos de derechos colectivos: el derecho al desarrollo.

\section{3.- NECESIDADES DE AUTOREALIZACIÓN, DERECHO AL DESARROLLO Y AGENDA 2030}

El derecho al desarrollo entendido como una pretensión generalizada desde el consenso de la comunidad internacional, plasmado en la Declaración de 1986, en la cual los Estados signatarios asumen el compromiso de garantizar un nivel de vida adecuado como una meta alcanzable en términos de mínimos, es decir, la vida digna de todas las personas debe partir de umbrales en los que mida "la dignidad de la vida" de la gente.

El papel de los indicadores en cuanto a su uso, adquiere mayor relevancia cuando el derecho al desarrollo surge en el escenario del Derecho Internacional de los Derechos Humanos. Puede intuirse, de acuerdo al contenido de la declaración, que el derecho al desarrollo, no es más que la garantía plena de los derechos económicos, sociales y culturales para todas las personas. Esto es puesto a consideración, toda vez que en términos de justiciabilidad estos derechos suponen ser un reto importante, puesto que son "costosos"25. La libertad negativa a diferencia de la libertad positiva, no supone más que la no intervención del Estado en la propia autodeterminación de las personas, garantizando su libertad ${ }^{26}$.

La libertad positiva, en cambio, requiere de condiciones mucho más operativas por las entidades del Estado, en este ámbito, debe ser garante y remover los obstáculos para que las personas obtengan sus derechos económicos, sociales y culturales. Es por ello que se les conocen como aquellos derechos que propenden por la igualdad de las personas, interviniendo para subsanar aquellas desventajas artificiales que existen entre unos y otros ${ }^{27}$. Tal igualdad entonces, no implica en ser subjetiva, en que todas las personas son homogéneas y que sus particularidades deban ser desconocidas por el Estado. Sino que las diferencias que son propias de los individuos, por razones de etnia, sexo, condición socioeconómica, entre otras, no deben suponer un criterio necesario de exclusión para el goce efectivo de los derechos.

Por lo tanto, la igualdad que pretenden los derechos económicos, sociales y culturales es objetiva, en cuanto a la relación

25 ARAGÓN RIVERA, Álvaro. Ciudadanía y derechos sociales: las dificultades de la ciudadanía social. Andamios, 2012, vol. 9, no 18, p. 144.

26 ÁlVAREZ, Silvina. La autonomía personal y la perspectiva comunitarista. Isegoría, 1999, no 21, p. 80.

27 CARTER, Ian. Libertad negativa y positiva. Óp., cit., p, 26. 
de la persona frente al acceso a las oportunidades que supone el ejercicio de los derechos sociales ${ }^{28}$. Esta naturaleza se encuentra relacionada con la interacción que tienen las personas con sus semejantes, cuando se trasciende del plano individual al plano colectivo, esto supone una relación de interdependencia para con los otros y que el goce de sus derechos no dependa solamente de la no intervención del Estado, sino del rol activo que este tenga en la intervención en términos de igualdad para fomentar la articulación entre diversos actores sociales en fomentar la inclusión de unos y otros en el "paraguas" del nivel de vida adecuado que otorga la igualdad de oportunidades.

Los Objetivos de Desarrollo Sostenible tienen como antecedente cercano los Objetivos de Desarrollo del Milenio, cuya diferencia parte de su ámbito de aplicación, mientras que los actuales son de ámbito universal, en cambio los ODM planteaban necesidades urgentes en "países en desarrollo". El ámbito universal de los ODS refuerza la teoría aquí planteada que estas metas a alcanzar por la comunidad internacional, contemplan el derecho al desarrollo como pretensión universal que involucra a todos los Estados.

Las necesidades de autorrealización planteadas por Maslow, como la cúspide del proyecto de vida planteado por el individuo, pueden ser asimiladas en el Derecho Internacional de los Derechos Humanos como la completa garantía de los derechos civiles y políticos y los derechos económicos sociales y culturales para todas las personas. Son entonces, el estado ideal de cobertura de derechos planteada en un Estado de bienestar.

Los ODS, pueden dividirse en los siguientes ámbitos vistos desde una perspectiva finalista, en cuanto al bien jurídico que se pretende proteger mediante su regulación:

\section{Tabla 3. Ejes temáticos Objetivos de Desarrollo Sostenible ${ }^{29}$}

\begin{tabular}{|l|l|}
\hline Eje temático & Objetivo de Desarrollo Sostenible \\
\hline \multirow{2}{*}{ Erradicación de la pobreza } & $\begin{array}{l}\text { ODS 1: Fin de la pobreza } \\
\text { ODS 2: Hambre cero } \\
\text { ODS 3: Salud y Bienestar }\end{array}$ \\
\hline Acceso a servicios básicos & $\begin{array}{l}\text { ODS 6: Agua limpia y saneamiento } \\
\text { ODS 7: Energía asequible y no contaminante }\end{array}$ \\
\hline $\begin{array}{l}\text { Oportunidades para el } \\
\text { desarrollo }\end{array}$ & $\begin{array}{l}\text { ODS 4: Educación de calidad } \\
\text { ODS 8: Trabajo decente y crecimiento económico }\end{array}$ \\
\hline $\begin{array}{l}\text { Fomentar la innovación y } \\
\text { estructuras resilientes }\end{array}$ & $\begin{array}{l}\text { ODS 9: Industria, innovación e infraestructura } \\
\text { ODS 11: Ciudades y comunidades sostenibles } \\
\text { ODS 12: Producción y consumo responsables }\end{array}$ \\
\hline Reducir las desigualdades & ODS 5: Igualdad de género \\
\hline
\end{tabular}

${ }^{28}$ SANCHÍS, Luis Prieto. Los derechos sociales y el principio de igualdad sustancial. Revista del centro de estudios constitucionales, 1995, no 22, p. 17.

${ }^{29}$ Fuente: Elaboración propia, con información de: Naciones Unidas, Agenda 2030: Objetivos de Desarrollo Sostenible. Convenciones: ODS, Objetivos de Desarrollo Sostenible. 


\begin{tabular}{|l|l|}
\hline & ODS 10: Reducción de las desigualdades \\
\hline \multirow{3}{*}{ Cuidado del ambiente } & ODS 13: Acción por el clima \\
& ODS 14: Vida submarina \\
& ODS 15: Vida de ecosistemas terrestres \\
\hline $\begin{array}{l}\text { Colaboración entre los } \\
\text { diferentes actores }\end{array}$ & ODS 16: Paz, justicia e instituciones solidas \\
ODS 17: Alianzas para lograr los objetivos
\end{tabular}

De los anteriores objetivos sistematizados en bloques temáticos al menos 11 de ellos pueden ser categorizados como Derechos Humanos desde la siguiente manera:

\section{Tabla 4. Objetivos de desarrollo sostenible que contienen derechos humanos 30}

\begin{tabular}{|c|c|}
\hline ODS & Tratados de Derechos Humanos \\
\hline ODS 1 & Art 25 (DUDH) y Art 11 (PDESC): Derecho a un nivel de vida adecuado \\
\hline ODS 2 & Art 3 (DUDH) y Art 6 (PDCyP): Derecho a la vida \\
\hline $\begin{array}{l}\text { ODS } 3 \\
\text { ODS } 6^{31} \\
\text { ODS } 7^{32}\end{array}$ & $\begin{array}{l}\text { Art } 12 \text { (PDESC): Derecho al disfrute del más alto nivel posible de salud } \\
\text { física y mental. }\end{array}$ \\
\hline ODS 4 & Art 26 (DUDH) y Art 13 (PDESC): Derecho a la educación. \\
\hline $\begin{array}{l}\text { ODS } 5 \\
\text { ODS } 10\end{array}$ & $\begin{array}{l}\text { Art } 1 \text { (DUDH): Todos los seres humanos nacen libres e iguales. } \\
\text { Art } 2 \text { (DUDH) y Art } 26 \text { (PDCyP): Principio de no discriminación. } \\
\text { Art } 7 \text { (DUDH): Igualdad ante la Ley. } \\
\text { Art } 16(\text { DUDH), Art } 23 \text { (PDCyP) y Art } 10 \text { (PDESC): Igualdad en contraer } \\
\text { matrimonio. } \\
\text { Art } 2 \text { (PDCyP): Aplicación universal del pacto. } \\
\text { Art } 3 \text { (PDCyP) y Art } 3 \text { (PDESC): Igualdad entre hombres y mujeres. }\end{array}$ \\
\hline ODS 8 & Art 23 (DUDH) y Art 6, 7 y 9 (PDESC): Derecho al trabajo. \\
\hline ODS 16 & $\begin{array}{l}\text { Art } 8 \text { (DUDH): Derecho a un recurso efectivo. } \\
\text { Art } 9 \text { (DUDH): Nadie podrá ser detenido arbitrariamente. } \\
\text { Art } 10 \text { (DUDH): Derecho a un juicio justo. } \\
\text { Art } 11 \text { (DUDH): Presunción de inocencia. } \\
\text { Art } 21 \text { (DUDH): Derecho a participar en la vida política. } \\
\text { Art } 4 \text { (PDCyP) y Art } 4 \text { (PDESC): Respeto de derechos en estados de } \\
\text { excepción. } \\
\text { Art } 7 \text { (PDCyP): Prohibición de torturas, tratos crueles, inhumanos y } \\
\text { degradantes. } \\
\text { Art } 9 \text { (PDCyP): Prohibición de detenciones arbitrarias. } \\
\text { Art } 10 \text { (PDCyP): Trato humano a las personas privadas de la libertad. } \\
\text { Art } 14 \text { (PDCyP): Igualdad ante los tribunales y cortes de justicia } \\
\text { Art } 15 \text { (PDCyP): Principio de legalidad en el derecho penal. }\end{array}$ \\
\hline ODS 17 & Art 28 (DUDH): Orden internacional para la efectividad de derechos. \\
\hline
\end{tabular}

30 Fuente: Elaboración propia con información de Naciones Unidas (Objetivos de desarrollo sostenible, Pacto de derechos Civiles y Políticos, Declaración Universal de Derechos Humanos y Pacto de Derechos Económicos, Sociales y Culturales). Convenciones: DUDH (Declaración Universal de Derechos Humanos), PDCyP (Pacto de Derechos Civiles y Políticos), PDESC (Pacto de Derechos Económicos, Sociales y Culturales).

31 Vinculadas al saneamiento básico como condición para conservar el disfrute del más alto nivel posible de salud física y mental.

32 Ibid. 
La relación existente entre los ODS y los derechos ya reconocidos en la práctica jurídica internacional, se encuentra en su interdependencia al igual que la de aquellos actores que componen la dinámica de protección de derechos, entre los cuales destacan las personas, organizaciones y los Estados ${ }^{33}$. La articulación en cada una de sus funciones hará que el desarrollo sostenible impacte en cada vez más escenarios, tal como se contemplan en los 17 objetivos. La declaración del derecho al desarrollo, contempla la cualidad de la interdependencia de los derechos que en su conjunto traen como consecuencia el mejoramiento del nivel de vida de todas las personas.

De los 17 ODS, 11 regulan derechos humanos, esto denota la prioridad que tiene para la comunidad internacional la universalidad del goce mayor posible de derechos para las personas. Como bien se mencionó anteriormente, una de las características principales de estos objetivos es su carácter universal fijando la sostenibilidad como el vector del desarrollo de los Estados. La eficacia de las necesidades de autorrealización fijadas en los ODS tomando al Desarrollo como meta a alcanzar mediante la garantía de los Derechos Sociales la cual, depende en gran parte de su justiciabilidad y de su efectiva prestación por parte del Estado. Esto, siempre será acorde a la capacidad de las instituciones para poder efectuar las políticas conducentes para su realización.

Resulta entonces, un desafío materializar el derecho al desarrollo (consecuencia de la garantía de los derechos consagrados en el Pacto de Derechos Civiles y Políticos y el Pacto de Derechos Económicos, Sociales y Culturales), el cual resulta muchas veces propio de los Estados con la suficiente capacidad económica para poder prestarlos a sus ciudadanos. Tal como lo enuncia Maslow, al momento de que el individuo tiene sus necesidades radicales y sociales satisfechas, entra al ámbito de las necesidades de autorrealización. De la misma manera ocurre con los Estados, mediante la superación de la pobreza y el haber alcanzado un nivel de vida adecuado, se plantea el desarrollo como una realidad para sus ciudadanos.

\section{4.- CONCLUSIONES}

Desde una perspectiva sistémica, las necesidades analizadas desde la pirámide de Maslow, partiendo de las más intensas o cercanas a la subsistencia del individuo, hasta las más prescindibles (en relación a la escasez de recursos para su satisfacción). La relación entre las necesidades y derechos fundamentales, más allá de las teorías capacitistas que se oponen a su fundamento teórico como

\footnotetext{
${ }^{33}$ ORDOÑEZ BALANZAIRO, Miriam. La nueva agenda de desarrollo post-2015: Los ODS y ¿La seguridad humana? Revista Internacional de Cooperación y Desarrollo, 2015, vol. 2, no 1, p. 68.
} 
las afirmadas por Martha Nussbaum, en su libro "Las fronteras de la justicia", encuentran su fundamento jurídico en la práctica jurídica internacional, que actúan como evidencia empírica de lo anteriormente planteado.

Las relaciones sociales son clave para que todo derecho sea realizable, este debe ser alcanzado es decir, se necesita de la intervención de muchas personas, que en el ejercicio de ciertas acciones contribuyan a la satisfacción de esa necesidad. Entonces, la respuesta del Estado ante tal situación, se hace mediante la configuración del derecho subjetivo.

Esto se contrasta con lo planteado por la tesis de la libertad negativa, en la que se fundamenta el liberalismo clásico, por la cual, el llamado es a permitir un campo de no intervención en la vida del individuo. De manera complementaria, la sociedad está llamada mediante sus instituciones (familia, Estado, etc.), a intervenir de acuerdo en favor del individuo en cada una de las fases de su vida mediante los cuidados.

El argumento que se suele utilizar en contra de que las necesidades fundamenten derechos, es en la definición de estas como hechos meramente descriptivos que no son normativas en sí mismas, por carecer de un valor "moral". Sin embargo, en contraposición a lo anterior, se puede fundamentar el valor moral de las necesidades en que estas tengan ese carácter imperativo de ser satisfechas, a través de la interdependencia como característica de los seres humanos. Siendo así, como lo enuncian los profesores Gregorio Peces Barba ${ }^{34}$ e Ignacio Campoy ${ }^{35}$, la solidaridad, la ética pública de la modernidad.

Para que las necesidades se encuentren satisfechas, siempre se va a depender en cierta medida que otras personas la hagan o dejen de hacer cosas en favor del colectivo y que esto repercuta en un beneficio individual masivo. Esto sustenta la necesidad de que exista una organización social que las satisfaga, y que esta cuente con la intervención por acción y por omisión de personas e instituciones en ciertos aspectos que se vean relacionados con las necesidades, y el imperativo institucional mediante la norma jurídica en la positivización por parte del Estado, actúa como agente que reconoce la existencia de derechos subjetivos.

Lo que se analiza en el presente artículo es una efectividad escalonada de los derechos subjetivos, teniendo en cuenta las necesidades del individuo empezando por aquellas que son radicales o cercanas su necesaria subsistencia. Luego los derechos sociales en la interacción de unos y otros actores en beneficio de terceros, para

\footnotetext{
34 PECES-BARBA MARTINEZ, Gregorio. La Dignidad Humana, Madrid, Ed. Dykinson, 2007, pp, 158 y 165.

35 CAMPOR CERVERA, Ignacio, "Una revisión de la idea de dignidad humana de los valores de libertad, igualdad y solidaridad en relación con la fundamentación de lo derechos", óp., cit., pp, 160-166.
} 
finalmente, satisfacer los derechos de autorrealización contenidos en el derecho al desarrollo y agenda 2030.

Los derechos sociales, siempre han sido vistos como un desafío en cuanto a su realización, por lo "costosos" que suponen. Más allá de la erogación presupuestaria que se deba realizar para su garantizar su efectividad, también debe considerarse la solidaridad como valor con efecto vinculante, no sólo en la expedición de normativa, sino en la ejecución de políticas públicas y fundamento de la función pública.

\section{5.- BIBLIOGRAFÍA}

ALEXY, Robert, Teoría de los derechos fundamentales, trad. del alemán de E. Garzón Valdez, Madrid, Centro de Estudios Constitucionales, 1993.

ÁLVAREZ, Silvina. La autonomía personal y la perspectiva comunitarista. Isegoría, 1999, no 21, p. 80.

AÑóN, María José. Ciudadanía social: La lucha por los derechos sociales. Cuadernos electrónicos de filosofía del derecho, 2002, vol. 6, p. 1-15.

ARAGÓN RIVERA, Álvaro. Ciudadanía y derechos sociales: las dificultades de la ciudadanía social. Andamios, 2012, vol. 9, no 18, p. 144.

ATRIA, F., "¿Existen derechos sociales?", Discusiones, La Pampa, Universidad Nacional del Sur, año 4, núm.4, 2005, pp. 15-59.

BACIGALUPO, Mariano. La aplicación de la doctrina de los «Limites Inmanentes» a los derechos fundamentales sometidos a reserva de limitación legal (A propósito de la sentencia del Tribunal Administrativo Federal alemán de 18 de octubre de 1990). Revista española de derecho constitucional, 1993, no 38, p. 297-315.

BÖCKENFÖRDE, Ernst-Wolfgang, Escritos sobre derechos fundamentales, trad. de Juan Luis Requejo e Ignacio Villaverde, Baden-Baden, Nomos, 1993, p. 95.

CAMPOY CERVERA, Ignacio. "Una revisión de la idea de dignidad humana de los valores de libertad, igualdad y solidaridad en relación con la fundamentación de lo derechos", Anuario de filosofía del derecho, No. 21, 2004, pp, 143-166.

CARTER, Ian. "Libertad negativa y positiva. Astrolabio: revista internacional de filosofía", 2010, no 10, p. 15-35.

CRUZ PARCERO, J., El lenguaje de los derechos. Ensayo para una teoría estructural de los derechos, Madrid, Trotta, 2007, pp. 7176. Madrid, 1989.

DWORKIN, Ronald, "Los derechos en serio", Ariel Derecho,

ESPINO TAPIA, Diana Rocío. "Derechos sociales y justiciabilidad en la teoría constitucional de inicios del siglo XXI", Cuestiones Constitucionales: Revista Mexicana de Derecho Constitucional, Núm. 36. 2017, pp: 83-104. 
FARRELL, Martín Diego. Libertad negativa y libertad positiva. Revista del Centro de Estudios Constitucionales, 1989, no 2, p. 9-20. GIMÉNEZ, Gilberto. Apuntes para una teoría de la identidad nacional. Sociológica México, 2015, no 21, p, 2.

GRASSETTI TONELLI, Eduardo. Satisfacción de necesidades y modelo social. PSOCIAL, 2018, vol. 4, no 1, p. 17.

IOSA, Juan. "Libertad negativa, autonomía personal y constitución", Revista Chilena de Derecho, vol. 44 No 2, 2017, p, 497. MASLOW, A.H."A theory of human motivation". Psychological Review, 50, 1943.370-396.

MERCADO MALDONADO, Asael; GONZÁLEZ VELÁSQUEZ, Guillermo. La teoría del conflicto en la sociedad contemporánea. Espacios públicos, 2008, vol. 11, no 21.

ORDOÑEZ BALANZAIRO, Miriam. La nueva agenda de desarrollo post-2015: Los ODS y ¿La seguridad humana? Revista Internacional de Cooperación y Desarrollo, 2015, vol. 2, no 1.

PECES-BARBA MARTINEZ, Gregorio. La Dignidad Humana, Madrid, Ed. Dykinson, 2007.

PISARELLO, G., Los derechos sociales y sus garantías. Elementos para una reconstrucción, Madrid, Trotta, 2007, pp. 59.

PUIG LLOBET, Montserrat; SABATER MATEU, Pilar; ÁVILA RODRÍGUEZ, Nuria. Necesidades humanas: evolución del concepto según la perspectiva social. Aposta. Revista de Ciencias Sociales, 2012, no 54, p. 2.

SANCHÍS, Luis Prieto. Los derechos sociales y el principio de igualdad sustancial. Revista del centro de estudios constitucionales, 1995, no 22, p. 17.

SORIANO, Ramón. "Sociología del Derecho", Ariel Derecho, Madrid: 1997.

TOLE MARTÍNEZ, Julián, La teoría de la doble dimensión de los derechos fundamentales en Colombia. El estado de cosas inconstitucionales, un ejemplo de su aplicación. Cuestiones Constitucionales, 2006, pp-253-316 